\title{
Estimation and Cancellation of Transponder Distortions in Satellite Forward Links Using Memory Polynomials ${ }^{\S}$
}

\author{
Svilen Dimitrov* \\ German Aerospace Center (DLR), Satellite Networks Department, 82234 Wessling, Germany
}

\begin{abstract}
SUMMARY
In this paper, a practical realization of a non-linear equalizer with distortion cancellation at a satellite receiver is studied, using a memory polynomial model for channel estimation and distortion cancellation. Varying the memory depth and the non-linear order of the memory polynomial model, the packet-error rate (PER) performance of this non-linear equalizer is compared to the standard fractionally-spaced linear adaptive equalizer, as well as to a setup of the non-linear equalizer with ideal channel knowledge at the receiver. In a single-carrier transponder setup similar to the direct-to-home (DTH) broadcast DVB-S2X reference scenario, the improved receiver with linear and non-linear equalization and practical channel estimation is shown to consistently approach the PER performance of the setup with ideal channel knowledge when increasing the memory depth and the non-linear order. The improved receiver demonstrates superior performance as compared to the standard fractionally-spaced linear adaptive equalizer with up to 5.48-dB energy efficiency gain for 64-APSK for a practical memory polynomial setup. Furthermore, it enables the use of high-order modulation up to 256-APSK in this scenario, improving significantly the spectral efficiency of the air interface. Copyright (c) 2018 John Wiley \& Sons, Ltd.
\end{abstract}

Received ...

KEY WORDS: Satellite communications; non-linear distortion; equalization; memory polynomial; highorder modulation; energy efficiency.

\section{INTRODUCTION}

With the extension of the second-generation digital video broadcasting system for satellite broadcasting and unicasting (DVB-S2X) standard [1], high-throughput-satellite (HTS) systems advance towards more bandwidth-demanding applications and services, e.g., direct-to-home (DTH) $4 \mathrm{~K}$ television broadcasting or broadband interactive Internet protocol (IP) services for very-smallaperture terminals (VSATs), emerging markets for mobile satellite platforms, such as broadband connectivity on airplanes, cruise ships and trains, as well as professional applications. Transmission

\footnotetext{
${ }^{*}$ Correspondence to: German Aerospace Center (DLR), Satellite Networks Department, 82234 Wessling, Germany. Email: svilen.dimitrov@dlr.de

$\S$ This is the pre-peer reviewed version of the following article: Dimitrov S., Estimation and Cancellation of Transponder Distortions in Satellite Forward Links Using Memory Polynomials, Int. J. Satellite Commun. Netw., Apr. 2018, which has been published in final form at http:// dx. doi.org/10.1002/sat.1239. This article may be used for noncommercial purposes in accordance with Wiley Terms and Conditions for Self-Archiving.
} 
over satellite is known to suffer from linear and non-linear signal distortions due to limitations of the onboard satellite transponder. In the forward-link, for example, in order to maximize the spectral efficiency, state-of-the-art satellite communication systems resort to using a very low carrier roll-off and high symbol baud rates close to the maximum transponder bandwidth [2]. Due to imperfect magnitude and group-delay responses of the input-multiplexing (IMUX) and outputmultiplexing (OMUX) onboard filters, however, linear distortion is introduced in the form of intersymbol interference (ISI). In addition, due to the power limitation of the satellite and operation of the travelling wave tube amplifier (TWTA) close to saturation, waveforms with higher-order modulation and multiple amplitude rings, e.g., 256-APSK, suffer from non-linear distortion in the form of constellation warping and clustering at the receiver demodulator. Without adequate channel compensation techniques, these adverse effects can reduce the spectral efficiency gains in the air interface expected with the application of tighter roll-off factors, higher symbol rates, and higher modulation orders.

Non-linear compensation schemes include a number of pre-distortion techniques at the transmitter, such as signal pre-distortion [3], [4], [5] and data pre-distortion [3], [6], [7], [8], [9], [10]. Signal pre-distortion [3] is commonly used for amplifier linearization. A solution against spectral regrowth is band-limited signal pre-distortion [4], [5] which applies digital filters to the out-ofband components and compensates the effect of the non-linear device on the in-band portion of the signal. Data pre-distortion [3], [6], [7], [8], [9], [10] is applied to the constellation symbols prior to pulse shaping, and it preserves the signal spectrum. Static data pre-distortion [2], [3], [6], [9] is the state-of-the-art solution in DVB-S2X. It accounts for static nonlinearities in the channel, whereby the transmitted constellation is modified such that the received centroids are close to the original transmitted constellation. Dynamic data pre-distortion [6], [7], [8], [9], [10] compensates in addition the memory effects in the channel. It takes into account the current symbol, as well as symbols preceding and succeeding each symbol. A common approach to handle the ISI is equalization at the receiver [3], where knowledge of the channel, obtained for instance by channel estimation, is used to minimize the ISI. A fractionally-spaced adaptive linear equalizer has been introduced in the DVB-S2X receiver [2].

Pre-distortion at the transmitter has been shown to be an effective channel compensation technique for a multi-carrier transponder in [10]. However, in a single-carrier transponder, symbolbased equalization with non-linear distortion cancellation at the receiver has shown significantly better results [11]. Decision-directed equalization [12] applied at the receiver is known to provide very good performance in channels with memory. A turbo equalizer using soft information exchange with the low-density parity check (LDPC) decoder has been proposed for application over non-linear satellite channels in [13]. A novel symbol-based equalizer with non-linear distortion cancellation, introduced in [14], reduces the complexity of the receiver by using maximum likelihood (ML) demodulation with hard decision in the cancellation loop with only marginal degradation of packeterror rate (PER) performance [11] in the single-carrier satellite forward link. Both approaches assume perfect channel knowledge at the receiver for estimation and cancellation of the non-linear distortion. In [11], knowledge of the transponder characteristics is assumed at the receiver, i.e., measured magnitude and group delay responses of the IMUX/OMUX filters, as well as the input amplitude/output amplitude (AM/AM) and input amplitude/output phase (AM/PM) characteristics of the TWTA. While such information can be obtained before the commission of the satellite, diurnal 
changes in the responses of the analog filters and variation of the output back-off (OBO) in the satellite transponder require channel estimation at the receiver.

In this paper, symbol-based equalization with non-linear distortion cancellation is studied for application in the satellite receiver, using practical channel estimation based on a memory polynomial model. The PER performance of the receiver is evaluated for the single-carrier transponder setup similar to the DTH reference scenario specified in the DVB-S2X implementation guidelines [2] for a high baud rate of 34 MBaud and a low carrier roll-off factor of $5 \%$ in a 36MHz transponder. Modulation orders of 16-APSK, 32-APSK, 64-APSK, 128-APSK, and 256-APSK are simulated with a 3/4-rate LDPC code. A number of memory polynomial setups are evaluated, varying the memory depth and the non-linear order, to show the trade-off between PER performance and complexity. The PER performance of the standard fractionally-spaced adaptive linear equalizer is also simulated to provide reference for the improvements. In addition, the ideal performance of the non-linear equalizer is also included, assuming knowledge of the exact characteristics of the IMUX/OMUX filters and the TWTA at the receiver. The practical channel estimation based on the memory polynomial model is shown to consistently approach the PER performance of ideal channel knowledge at the receiver with the increase of the memory depth and the non-linear order. As compared to standard linear equalization, the non-linear equalizer enables the use of the higher modulation orders of 128-APSK and 256-APSK, and shows significant energy efficiency gains of, e.g., $5.48 \mathrm{~dB}$ for 64 -APSK, using a memory polynomial model with memory depth of 10 and nonlinear order of 5 . The non-linear equalizer is particularly suitable for application in waveforms with higher-order modulation, low roll-off factors and high symbol rates close to the limits of the transponder bandwidth, improving the spectral efficiency of the air interface.

The rest of the paper is organized as follows. Section 2 describes the satellite system model, including the transmission chain and the channel modelling. Section 3 describes the studied channel compensation algorithms at the receiver. Section 4 presents the performance evaluation results. Finally, Section 5 concludes the paper.

\section{SATELLITE SYSTEM MODEL}

In a satellite communication system, a gateway relays message signals designated to user terminals over a satellite. In this section, the satellite transmission chain in the forward link and the satellite channel model are presented.

\subsection{Satellite Transmission Chain}

The block diagram of the satellite transmission chain is presented in Fig. 1. At the gateway transmitter, a stream of data packets with 1504 bits per packet are encoded by means of a forward error correction (FEC) scheme, and modulated for transmission. In DVB-S2X [1], first, a Bose-Chaudhuri-Hocquenghem (BCH) encoder is applied, followed by an LDPC encoder and bit interleaver. The resulting FEC frame with length of up to 64800 bits is mapped to symbols, using APSK modulation with constellations up to 256-APSK at the modulator. The modulated symbols are grouped in physical-layer (PL) frames with up to 67920 symbols per PL frame. 


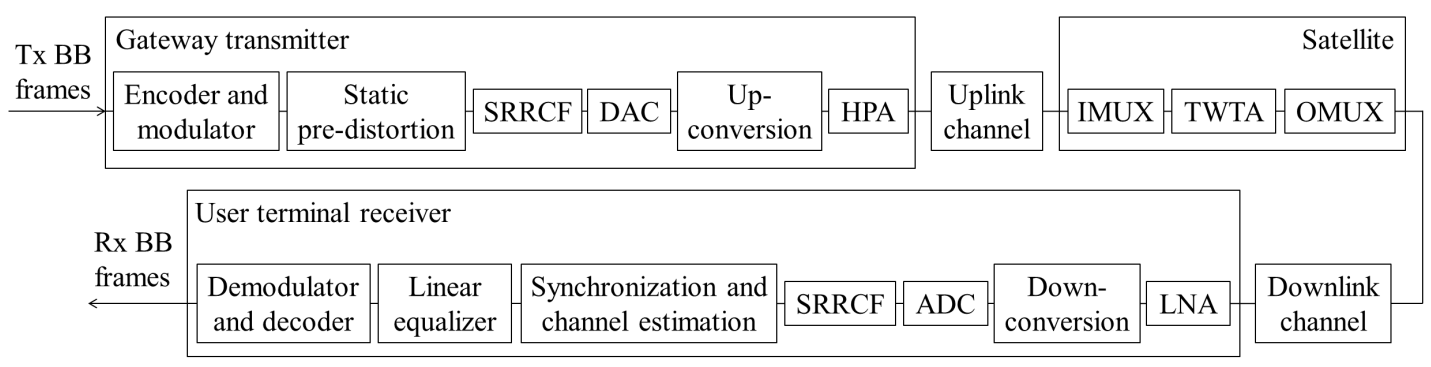

Figure 1. Block diagram of the satellite transmission chain in the forward link.

The DVB-S2X transmitter employs static data pre-distortion [2], [3], [6], [9] to compensate constellation warping effects due to non-linear distortion at the satellite transponder. The symbols are pulse shaped by means of a square root raised cosine filter (SRRCF), in order to ensure spectrum integrity of the waveform for RF transmission over the feeder link. In the analog front-end, after digital-to-analog conversion (DAC), the baseband signal is up-converted to the carrier frequency, e.g., in the Ka band. Finally, the signal is amplified by means of a high-power amplifier (HPA) at the gateway, and is transmitted over the feeder link through the uplink atmospheric channel.

At the transponder onboard the satellite, the signal is passed through an IMUX filter, is amplified by a TWTA, and is passed through an OMUX filter in order to guarantee spectrum integrity for transmission over the user link. Here, the imperfect frequency responses of the IMUX and OMUX filters introduce linear distortion in the form of ISI, while the TWTA introduces non-linear distortion. The signal is then transmitted over the downlink atmospheric channel.

At the user terminal receiver, the signal is amplified by means of an LNA, and is down-converted to baseband. Next, an analog-to-digital converter (ADC) and a matched SRRCF are applied. After timing, frame synchronization and channel estimation, the symbols are passed through a linear equalizer to counter the linear distortion. A fractionally-spaced adaptive linear equalizer with 42 complex-valued taps is applied, working at twice the symbol rate. The symbols are then downsampled to the Nyquist rate. Finally, the demodulator and the decoder recover the received data packets. Here, a soft demapper generates log-likelihood ratios (LLRs), which after deinterleaving are provided as input to the decoding stages, i.e., an LDPC decoder, followed by a BCH decoder.

A number of simplifications of the satellite transmission chain in the forward link can be considered, preserving all key aspects that affect the physical layer performance. The free-space signal propagation can be characterized as a slow-fading process due to rainy weather, and is handled by means of adaptive coding and modulation (ACM), as well as a fading margin in the link budget. The flat gain of the atmospheric satellite channel is also a factor in the link budget, included in the calculation of the available SNR at the receiver. Since the physical layer performance is evaluated as a function of the SNR, unity atmospheric channel gain is considered in the simulations presented in Section 4. Since the HPA at the gateway is operated in the linear regime, the nonlinearity in the satellite channel is introduced primarily by the TWTA. The transmitter and receiver are assumed to be fine synchronized, i.e., genie-aided synchronization is performed based on correlation with the transmitted signal, ideally assumed to be known. In general, the signal is also distorted by phase noise at the receiver due to local oscillator instabilities during frequency conversion. However, since fine phase compensation for higher-order modulation is still an open issue [2], [6], and the focus of 
this paper is on compensation of the linear and non-linear distortions of the satellite transponder, phase noise is not considered in the channel modeling.

As a result, the received PL frame at the input of the demodulator at the user terminal receiver can be expressed as:

$$
\mathbf{x}^{(0)}=\operatorname{Eq}\left\{\mathbf{h}_{\mathrm{SRRCF}} *\left[\mathbf{h}_{\mathrm{OMUX}} * F\left(\mathbf{h}_{\mathrm{IMUX}} * \mathbf{h}_{\mathrm{SRRCF}} * \operatorname{Pre}\{\mathbf{x}\}\right)+\mathbf{w}\right]\right\},
$$

where $\mathbf{x}$ is the transmitted symbol vector in baseband, $\operatorname{Pre}\{\cdot\}$ is the applied static pre-distortion operator, $\mathbf{h}_{\mathrm{SRRCF}}$ is the impulse response vector of the SRRCF filter, and $\mathbf{h}_{\mathrm{IMUX}}$ is the impulse response of the IMUX filter. The non-linear transfer function of the TWTA is denoted as $F(\cdot)$, $\mathbf{h}_{\text {OMUX }}$ is the impulse response of the OMUX filter, $\mathbf{w}$ is a vector with samples of additive white Gaussian noise (AWGN), and $\mathrm{Eq}\{\cdot\}$ is the linear equalizer operator. In this baseband model, the transmitted signal $\mathbf{x}$ has an average energy per symbol of $E_{\mathrm{s}}$, and the AWGN $\mathbf{w}$ has a power spectral density of $N_{0}$. In addition, the responses of the SRRCFs and the IMUX/OMUX filters are normalized for unity average power. The same applies for the pre-distorter and the equalizer. Finally, the amplifier function is normalized for unity peak power.

\subsection{Satellite Channel Modelling}

In the satellite forward link, the main sources of distortion are the imperfect characteristics of the IMUX and OMUX filters and the non-linear TWTA response. Measured magnitude and groupdelay responses of the IMUX and OMUX filters in the satellite transponder have been provided as a reference in the DVB-S2X implementation guidelines [2], as shown in Fig. 2. While the guidelines only specify the set of characteristics of a $36-\mathrm{MHz}$ transponder, the filter responses are equally applicable to a $225-\mathrm{MHz}$ transponder by using appropriately specified scaling factors for the frequency axis. The IMUX/OMUX filters introduce linear distortion in the form of memory effects due to frequency selectivity at high baud rates, which corrupts the matched filtering at the receiver and amplifies the AWGN.

In this study, a not-linearized TWTA is considered to evaluate the performance of the nonlinear equalizer in a highly non-linear channel. This is similar to the DTH scenario specified in the DVB-S2X implementation guidelines [2]. The AM/AM and AM/PM characteristics are presented in Fig. 3, and are denoted as $A(\cdot)$ and $\phi(\cdot)$, respectively. They only depend on the signal amplitude. As a result, the output signal after the TWTA, $F(s)$, can be expressed as $F(s)=A(|s|) \exp [j \phi(|s|)+j \arg (s)]$, where $s$ is the input signal.

In order to facilitate an efficient energy consumption onboard the satellite, it is desirable to operate the TWTA close to saturation. In this non-linear regime, signals from higher-order APSK constellations are known to suffer from non-linear distortions. Therefore, an OBO is applied to shift the power transfer towards the linear regime at the cost of a penalty in the energy efficiency. The OBO is defined as the ratio between the peak output power of the amplifier and the average output power of the signal in the linear domain [14]. The non-linear distortion introduced by the TWTA can be described as constellation warping and symbol clustering at the input of the demodulator at the receiver [6]. The interfering component is dependent on the signal waveform, and therefore contains useful information. In single-carrier digital transmission schemes, as well as in multicarrier transmission with a small number of carriers, the interfering component is correlated with 

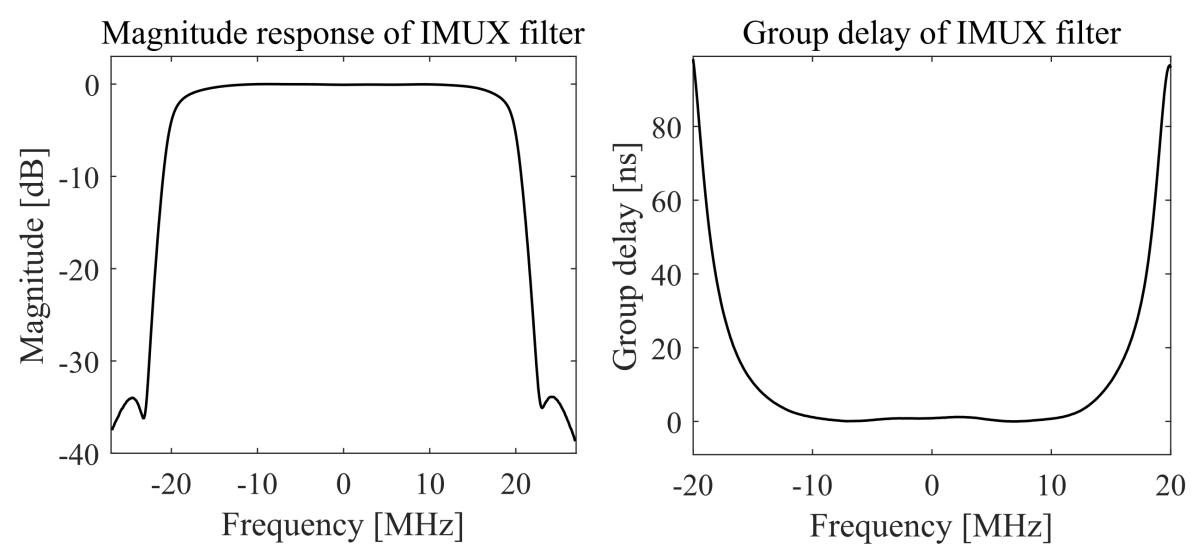

(a) Magnitude and group delay responses of IMUX filter.
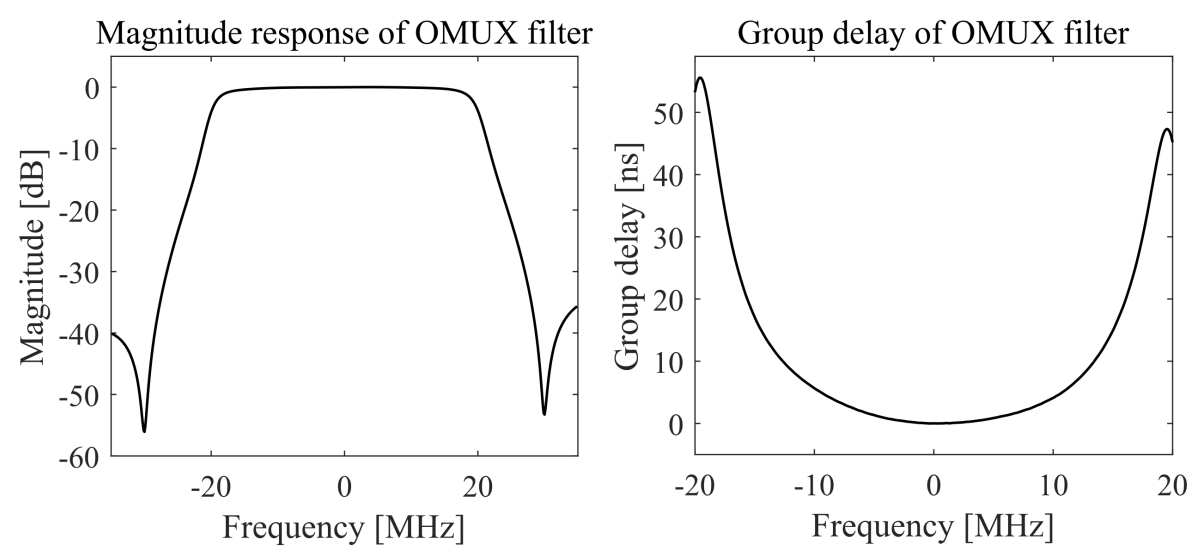

(b) Magnitude and group delay responses of OMUX filter.

Figure 2. Filter characteristics of a 36-MHz satellite transponder in the forward link.

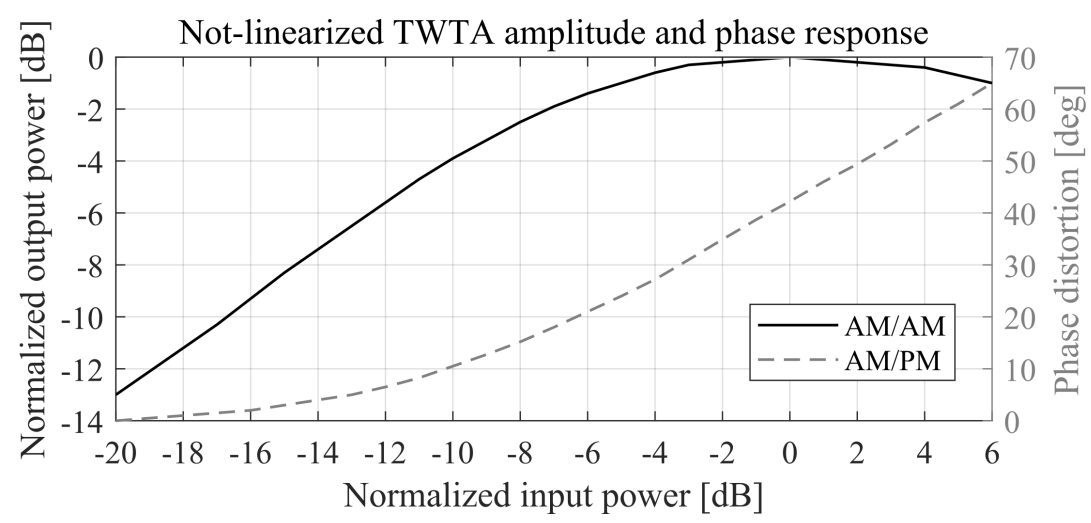

Figure 3. AM/AM and AM/PM transfer characteristics of a not-linearized TWTA.

the transmitted symbols. The received symbols can be decomposed in an attenuated and rotated replica of the transmitted symbols plus non-linear distortion noise. Each received centroid can be individually warped, i.e., it is scaled by a complex-valued factor relative to the transmitted 
constellation point. There is no general assumption on the distribution of the non-linear distortion noise.

\section{RECEIVER ALGORITHMS FOR CHANNEL COMPENSATION}

In this section, the state-of-the-art channel compensation algorithms at the receiver are discussed. A non-linear symbol-based equalizer performing distortion estimation and cancellation by means of a memory polynomial model is proposed as an addition to the fractionally-spaced adaptive linear equalizer specified in the DVB-S2X implementation guidelines [2]. The building blocks of the nonlinear equalizer, including the channel estimation and training blocks, are defined.

\subsection{State of the Art}

In literature, the non-linear compensation solutions include a variety of pre-distortion techniques at the transmitter, such as signal pre-distortion [3], band-limited signal pre-distortion [4], [5], and data pre-distortion [3], [6], [7], [8], [9]. In scenarios, where measured models of the transponder characteristics are unavailable or change over time, the pre-distorter function can be computed adaptively using indirect learning [7], [8] or direct learning algorithms [8]. For this purpose, a model for the non-linear channel with memory is required, e.g., a Volterra series [15] or a memory polynomial model [7], [8], [16]. In data pre-distortion algorithms, these models include the linear and non-linear blocks along the chain, e.g., the pulse shaping SRRCF at the transmitter, the IMUX/OMUX filters, the TWTA, and the matched SRRCF at the receiver.

In order to compensate the linear distortion introduced by the imperfect magnitude and group delay responses of the IMUX/OMUX filters onboard the satellite, a fractionally-spaced adaptive linear equalizer with 42 taps working at twice the symbol rate has been introduced in DVB-S2X [2]. In order to also compensate non-linear distortions, decision-directed detection by means of turbo equalization has been proposed for application in non-linear satellite channels in [13]. A channel model based on Volterra series is assumed, and minimum mean-squared error (MMSE) filters are computed to cancel the non-linear terms in an iterative fashion, using soft information exchange with the LDPC decoder. In [14], the symbol-based iterative equalization scheme has been introduced which reduces the complexity of the receiver by means of a simplified model for the received symbols after the non-linear device, and by using ML demodulation with hard decision in the cancellation loop. Instead of computing MMSE filters for every component in the Volterra series, a simple procedure can be applied to reconstruct and cancel the non-linear noise component. At the heart of the simplification is a first-order decomposition of the received symbols at the input of the demodulator, based on a symbol scaling factor representing the warping effect and a non-linear noise component containing the higher non-linear orders. In addition to non-linear compensation, memory effects in the form of ISI are also taken into account in the reconstruction and cancellation of the interfering component due to the fact that the equalizer operates on a large block of symbols, e.g., a PL frame. This equalizer has been shown to be an effective technique to compensate nonlinear distortions in the satellite channel, and to significantly reduce the SNR penalty with respect to the linear channel [14], [17], [18], [11]. 
In previous work [14], [17], [18], [11], known measured models for the distortion sources have been used in the estimation and cancellation of the non-linear noise performed by the symbol-based equalizer. These include the linear and non-linear distortions in the satellite transponder due to the IMUX filter, TWTA, and OMUX filter, such as the measured AM/AM and AM/PM characteristics of the TWTA and the measured magnitude and group delay responses of the IMUX and OMUX filters, obtained before the commission of each satellite. In this paper, the application of a memory polynomial model [7], [8], [16] is considered at the receiver, in order to estimate the non-linear channel with memory. As a result, the non-linear equalizer can compensate the slow time-variant changes in the transponder characteristics, such as diurnal changes in the responses of the analog filters or variations in the OBO.

The proposed equalizer estimates and cancels the interference in the non-linear channel with memory based on detection of the symbols at the receiver. In the satellite forward link, user terminal normally receives the signal on a single carrier, unless multiple tuners are assumed. As a result, it can only compensate the self-interference within that carrier, in the form of ISI, caused during the transmission over the non-linear channel with memory. Therefore, the proposed equalizer is best suitable for single-carrier scenarios in the satellite forward link. It can also be applied in a multicarrier scenario in combination with a pre-distortion technique at the transmitter that compensates the IMI. In this case, the proposed equalizer can further minimize the residual self-interference within the received carrier.

\subsection{Fractionally-spaced Adaptive Linear Equalizer}

In order to compensate the memory effects in the channel, an adaptive linear equalizer is applied at twice the symbol rate. The symbol column vector at the output of the linear equalizer can be expressed as $\mathbf{x}^{(0)}=\operatorname{Eq}\{\mathbf{y}\}=\mathbf{y} * \mathbf{e}^{T}$, where $\operatorname{Eq}\{\cdot\}$ is the equalizer operator, $*$ is the linear convolution operator, $(\cdot)^{\mathrm{T}}$ is the transpose operator, $\mathbf{y}$ is the symbol column vector at the input of the linear equalizer, and $\mathbf{e}$ is the row vector with the equalizer taps. These taps are updated in a data-aided manner, e.g., by using the following adaptive recursive algorithm [12]:

$$
\mathbf{e}_{l+1}=\mathbf{e}_{l}-\mu\left(\mathbf{e}_{l} \mathbf{y}_{l}^{\mathrm{T}}-x_{l}\right) \mathbf{y}_{l}^{*},
$$

where $\mathbf{e}_{l+1}$ is the updated equalizer tap vector after the $l$-th training symbol $x_{l}, \mathbf{e}_{l}=$ $\left[e_{0}, e_{1}, \ldots, e_{N_{\mathrm{EQ}}-1}\right]$ contains $N_{\mathrm{EQ}}$ taps, initialized as the zero vector $\mathbf{e}_{0}=[0, \ldots, 0]$. The window row vector containing $N_{\mathrm{EQ}}=42$ samples of the received symbol vector $\mathbf{y}$ is denoted as $\mathbf{y}_{l}=\left[y_{l}, y_{l-1}, \ldots, y_{l-N_{\mathrm{EQ}}}\right]$, and is initialized as $\mathbf{y}_{0}=\left[y_{0}, 0, \ldots, 0\right]$. Here, $(\cdot)^{*}$ denotes complex conjugation. With values between 0 and 1 , the step size $\mu$ represents a trade-off between convergence speed and stability, e.g., $\mu=0.001$.

\subsection{Non-linear Equalizer with Distortion Cancellation Using a Memory Polynomial Model}

In order to improve the performance of the receiver, a non-linear equalizer performing estimation and cancellation of the transponder distortions is added after the fractionally-spaced adaptive linear equalizer. The block diagram of the improved receiver is presented in Fig. 4. The symbols at the output of the linear equalizer, downsampled to the Nyquist rate, are provided as input to the iterative 


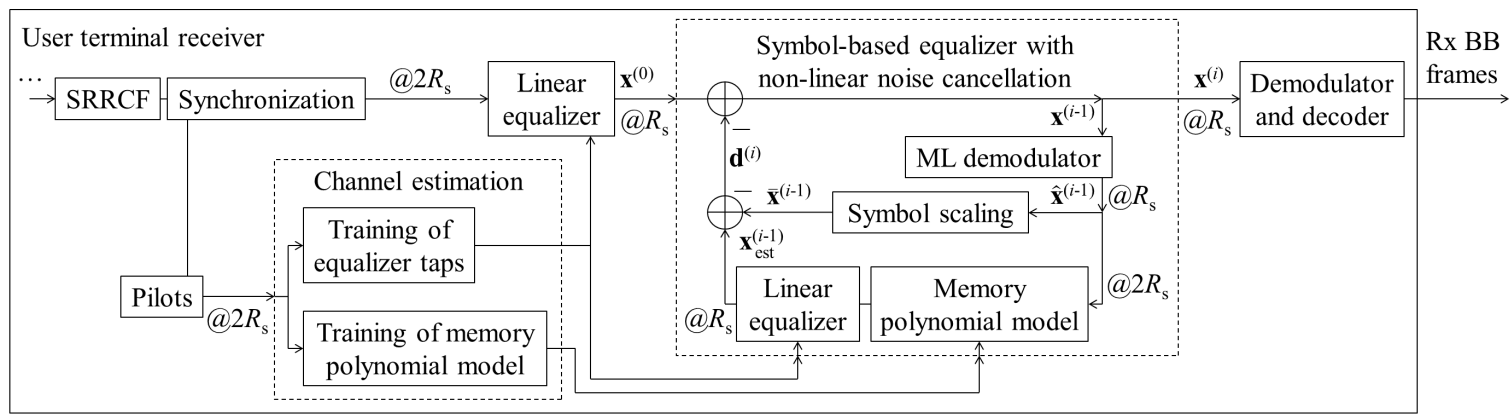

Figure 4. Block diagram of the proposed receiver using a symbol-based equalizer with non-linear distortion cancellation in the satellite forward link.

non-linear equalizer. At this point, a set of received symbols, e.g., a received PL frame $\mathbf{x}^{(0)}$, is buffered.

As a first step of the processing, detection is performed. The received symbols are ML demodulated by means of a hard decision using the received constellation centroids as a reference. The vector of detected symbols used in the $i$-th iteration is denoted as $\widehat{\mathbf{x}}^{(i-1)}=\operatorname{Demod}\left[\mathbf{x}^{(i-1)}\right]$.

The estimate of the interfering component is obtained through subtraction of two terms. To obtain the first term, the detected symbols are scaled by individual attenuation factors, $K_{c_{m}}$, according to the respective detected constellation point $c_{m}, m=1, \ldots, M$, with $M$ being the modulation order, to obtain $\overline{\mathbf{x}}^{(i-1)}$, where $\bar{x}_{c_{m}}^{(i-1)}=K_{c_{m}} \widehat{x}_{c_{m}}^{(i-1)}$. This is essential to account for the constellation warping effects. The individual scaling factors represent the ratio of a received centroid to the corresponding transmitted constellation point.

To obtain the second term, the detected symbols $\widehat{\mathbf{x}}^{(i-1)}$ are used to estimate the distorted received symbols using the memory polynomial model [7], [8], [16]:

$$
y(n)=\mathcal{M}\{x(n)\}=\sum_{k=1}^{K} \sum_{q=0}^{Q} a_{k q} x(n-q)|x(n-q)|^{k-1},
$$

where $x(n)$ is the input sequence with length of $N$ symbols, $n=0, \ldots, N-1, y(n)$ is the output sequence, $K$ is the non-linear order, $Q$ is the memory depth, and $a_{k q}$ is the complex-valued coefficient associated with the term with non-linear order $k, k=1, \ldots, K$, at time $q, q=0, \ldots, Q$. In practice, only the odd non-linear orders are sufficient to describe the nonlinearity. Similarly to the fractionally-spaced adaptive linear equalizer, the memory polynomial model is trained at twice the symbol rate.The training can be performed, e.g., by solving the indirect learning problem [7] for the vector a:

$$
\mathbf{y}=\mathbf{U a}
$$

where $\quad \mathbf{y}=[y(0), \ldots, y(N-1)]^{T}, \quad \mathbf{a}=\left[a_{10}, \ldots, a_{K 0}, \ldots, a_{1 Q}, \ldots, a_{K Q}\right]^{T}, \quad \mathbf{U}=\left[\mathbf{u}_{10}, \ldots, \mathbf{u}_{K 0}, \ldots\right.$, $\left.\mathbf{u}_{1 Q}, \ldots, \mathbf{u}_{K Q}\right], \mathbf{u}_{k q}=\left[u_{k q}(0), \ldots, u_{k q}(N-1)\right]^{T}$, and $u_{k q}(n)$ is defined as:

$$
u_{k q}(n)=x(n-q)|x(n-q)|^{k-1} .
$$


The least squares (LS) solution is given as:

$$
\widehat{\mathbf{a}}=\left(\mathbf{U}^{H} \mathbf{U}\right)^{-1} \mathbf{U}^{H} \mathbf{y},
$$

and it can be used to define:

$$
\mathcal{M}\{\mathbf{x}\}=\mathbf{U} \widehat{\mathbf{a}},
$$

After application of the already trained equalizer operator, the estimate of the received symbols can be expressed as:

$$
\mathbf{x}_{\text {est }}^{(i-1)}=\operatorname{Eq}\left\{\mathcal{M}\left\{\widehat{\mathbf{x}}^{(i-1)}\right\}\right\} .
$$

The scaled symbols are subtracted from the estimate of the received symbols to reconstruct an estimate of the non-linear distortion noise as:

$$
\mathbf{d}^{(i)}=\mathbf{x}_{\mathrm{est}}^{(i-1)}-\overline{\mathbf{x}}^{(i-1)}
$$

This estimate of the interfering component is subtracted from the buffered symbols to obtain a new set of the received symbols as output of the first iteration of non-linear distortion cancellation. In the second iteration, the newly obtained received symbols are detected and used to better estimate the interfering component, which is again subtracted from the originally buffered received symbols. The improved vector of received symbols after the $i$-th iteration can be expressed as:

$$
\mathbf{x}^{(i)}=\mathbf{x}^{(0)}-\mathbf{d}^{(i)}
$$

The output of this process are the newly obtained received symbols after a number of iterations. Finally, the buffer is released and a new frame of received symbols is processed. As the iterative symbol-based equalizer outputs symbols with reduced interference, more reliable LLRs can be computed at the subsequently applied soft demapper, improving the performance of the decoder.

\section{PERFORMANCE EVALUATION}

In this section, PER performance of the non-linear equalizer applied at the receiver is studied for application in the satellite forward link. The satellite channel model consists of the IMUX/OMUX filter responses and the transfer characteristic of the TWTA given in Section 2.2. A singlecarrier scenario is considered as representative of state-of-the-art satellite broadcast DTH networks, specified as one of the four reference scenarios in the DVB-S2X implementation guidelines [2]. Constellations of 16-APSK, 32-APSK, 64-APSK, 128-APSK, and 256-APSK are simulated, whereby one modulation order is applied per PL frame, in conjunction with a 3/4-rate LDPC code. A carrier symbol rate of $34 \mathrm{MBaud}$ with $5 \%$ roll-off factor is considered as an example of high carrier symbol rate with low roll-off factor in the transponder bandwidth of $36 \mathrm{MHz}$, as used in the DTH performance evaluation in [2]. In addition, non-linear distortion is added by operating the TWTA at low OBO settings. The optimum OBO values of $1.6 \mathrm{~dB}$ and $2.3 \mathrm{~dB}$ for 16-APSK and 32-APSK, respectively, are borrowed from the single-carrier DTH scenario in [2], and expanded as $3 \mathrm{~dB}, 3.7 \mathrm{~dB}$, and $4.5 \mathrm{~dB}$ for 64 -APSK, 128-APSK, and 256-APSK, respectively. 
The PER performance of the fractionally-spaced adaptive linear equalizer, applied as a standard feature of the DVB-S2X receiver, is presented as a reference scenario. The proposed non-linear equalizer is applied immediately after the linear equalizer. For estimation of the non-linear channel with memory, the memory polynomial model is applied with a number of combinations of memory and non-linear order parameters: 1) highest non-linear order of $K=3$ and memory of $Q=4$ taps, 2) $K=5$ and $Q=6,3) K=5$ and $Q=10,4) K=7$ and $Q=20$. Here, only the odd non-linear orders are used in the memory polynomial model. In addition, the ideal performance of the non-linear equalizer is simulated assuming knowledge of the exact characteristics of the IMUX/OMUX filters and the TWTA at the receiver. These scenarios exhibit the trade-offs between PER performance, complexity, and practicality. While more practical, channel estimation by means of a memory polynomial model has inferior PER performance as compared to perfect channel knowledge at the receiver. In addition, the considered scenarios for memory depth and nonlinear order of the memory polynomial models reveal the trade-off between PER performance and complexity of the channel estimation. The PER performance of the non-linear equalizer is evaluated for 2 iterations of non-linear distortion cancellation, shown to be provide sufficient convergence in [11].

The performance of the channel compensation techniques at the receiver is evaluated by means of a Monte Carlo simulation of the PER in the satellite forward link. The PER is evaluated as a function of the SNR defined as $P_{\text {sat }} / N$, i.e., the ratio of the onboard TWTA saturation power to the noise power, which can be related to the $E_{\mathrm{s}} / N_{0}$ ratio in the linear domain as follows [2]:

$$
\frac{P_{\mathrm{sat}}}{N}=\frac{E_{\mathrm{s}}}{N_{0}} \frac{R_{\mathrm{s}}}{W_{\text {ref }}} \mathrm{OBO},
$$

where $R_{\mathrm{S}}$ is the symbol rate and $W_{\text {ref }}$ is the transponder bandwidth in single-carrier mode. A high number of long PL frames are simulated, e.g., up to $10^{6}$ packets per simulation point, resulting in sufficient convergence of the statistics down to a PER of $10^{-3}$.

The PER performance results are presented in Figs. 5, 6, 7, 8, and 9 for the respective modulation orders. In addition, the SNR requirements at PER of $10^{-3}$ are summarized in TABLE I. The presented results of the reference linear equalizer in this paper are comparable with the results reported in [2] in the single-carrier DTH scenario with 34-Mbaud symbol rate and 5\% roll-off for 3/4-rate 16-APSK and 32-APSK. The standard linear equalizer is unable to compensate the transponder distortions for the higher modulation orders of 128-APSK and 256-APSK, and the PER performance saturates. The ideal performance of the non-linear equalizer reveals SNR gains of up to $1.79 \mathrm{~dB}$ for 16-APSK, $3.19 \mathrm{~dB}$ for 32-APSK, and $8.45 \mathrm{~dB}$ for 64-APSK. In addition, the 128-APSK and 256-APSK are enabled for practically achievable SNRs of $17.37 \mathrm{~dB}$ and $19.7 \mathrm{~dB}$, respectively. The use of the memory polynomial model for channel estimation is shown to approach the PER performance of perfect channel knowledge at the receiver with the increase of the model complexity, consistently across all modulation orders. While a non-linear order of up to $K=5$ shows to provide sufficient representation of the nonlinearity in the channel, a higher memory depth of up to $Q=10$ is required to obtain a sweet spot between PER performance and complexity. The memory polynomial models with lower memory depths of $Q=4$ and $Q=6$ show considerable improvements of the SNR requirement of up to $4.31 \mathrm{~dB}$ for 64 -APSK, and they manage to enable 128-APSK at $26.64 \mathrm{~dB}$ and $25.82 \mathrm{~dB}$, respectively. Using a memory depth of $Q=10$ increases the 


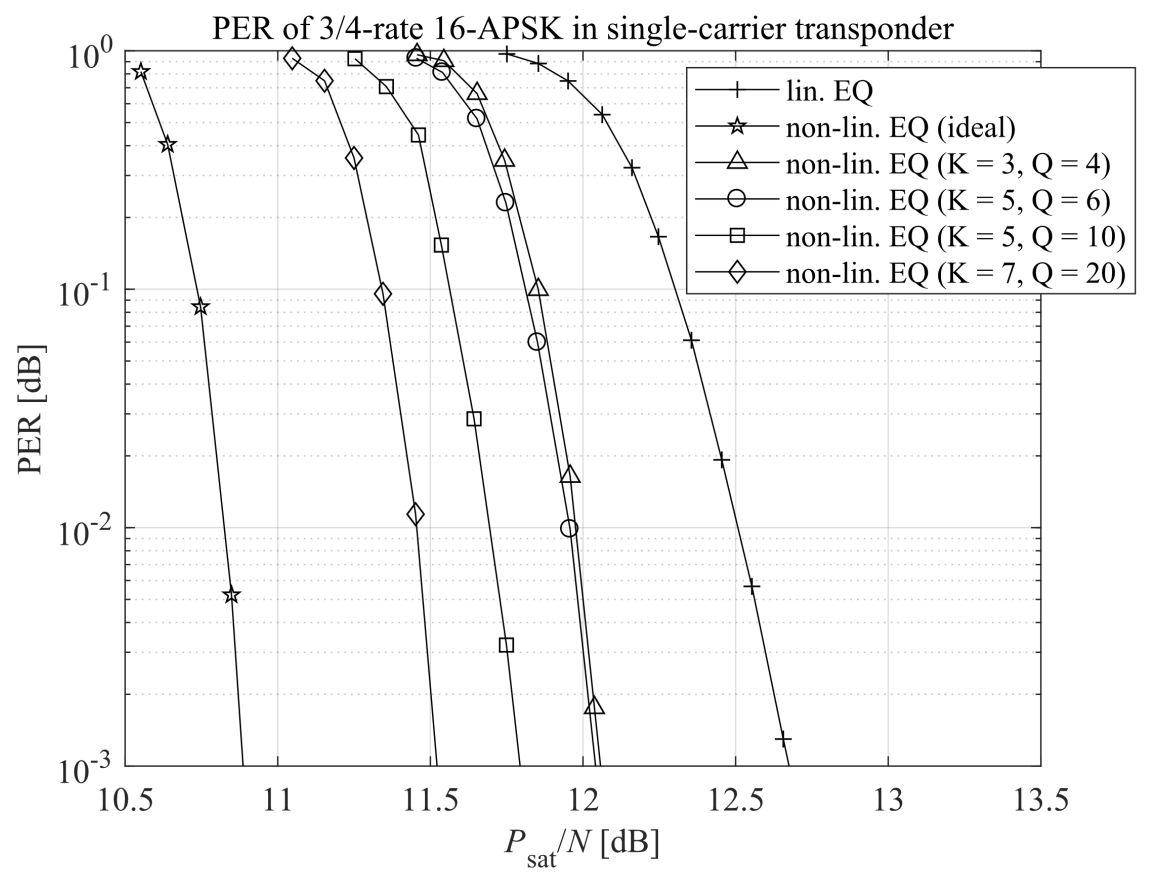

Figure 5. PER of 16-APSK modulation with 3/4-rate LDPC code in a single-carrier 34-Mbaud 5\%-roll-off signal in $36-\mathrm{MHz}$ forward link transponder. Standard linear equalization is compared with 2 iterations of non-linear equalization.

\begin{tabular}{|l|l|l|l|l|l|l|}
\hline $\begin{array}{l}\text { 3/4-rate } \\
\text { MODCODs }\end{array}$ & $\begin{array}{l}\text { Lin. } \\
\text { EQ [2] }\end{array}$ & $\begin{array}{l}\text { Non- } \\
\text { lin. EQ } \\
\text { (ideal) }\end{array}$ & $\begin{array}{l}\text { Non-lin. } \\
\text { EQ }(K=3, \\
Q=4)\end{array}$ & $\begin{array}{l}\text { Non-lin. } \\
\text { EQ }(K=5, \\
Q=6)\end{array}$ & $\begin{array}{l}\text { Non-lin. } \\
\text { EQ }(K=5, \\
Q=10)\end{array}$ & $\begin{array}{l}\text { Non-lin. } \\
\text { EQ }(K=7, \\
Q=20)\end{array}$ \\
\hline 16-APSK & 12.68 & 10.89 & 12.06 & 12.04 & 11.79 & 11.52 \\
\hline 32-APSK & 15.77 & 12.58 & 14.68 & 14.58 & 14.07 & 13.67 \\
\hline 64 -APSK & 23.41 & 14.96 & 19.31 & 19.1 & 17.93 & 17.06 \\
\hline 128-APSK & Saturates & 17.37 & 26.64 & 25.82 & 22.29 & 20.49 \\
\hline 256-APSK & Saturates & 19.7 & Saturates & Saturates & 30.68 & 24.69 \\
\hline
\end{tabular}

Table I. Required $P_{\mathrm{sat}} / N[\mathrm{~dB}]$ at $10^{-3}$ PER of the standard linear equalizer [2] in comparison to the additional non-linear equalizer with distortion cancellation at the receiver in a single-carrier satellite forward link at practical OBO.

gain for 64-APSK to $5.48 \mathrm{~dB}$. Furthermore, this setup reduces the SNR requirement of 128-APSK to $22.29 \mathrm{~dB}$, and even enables 256-APSK at $30.68 \mathrm{~dB}$. The presented considerable improvements in energy efficiency and the enabled use of higher-order modulation with high symbol rates close to the limits of the transponder bandwidth, as well as very low roll-off factors, show that the proposed non-linear equalizer is a practical solution to increase the spectral efficiency of the air interface, which translates into increased user data rates and satellite system capacity.

Considering the computational complexity of the receiver, the training of the memory polynomial model requires the inversion of a matrix in (6) with size of $(Q+1)(K+1) / 2 \times(Q+1)(K+1) / 2$, using only the odd non-linear orders. This training, however, is only performed sporadically, since the channel characteristics change only slowly over time, and therefore it can be performed offline. 


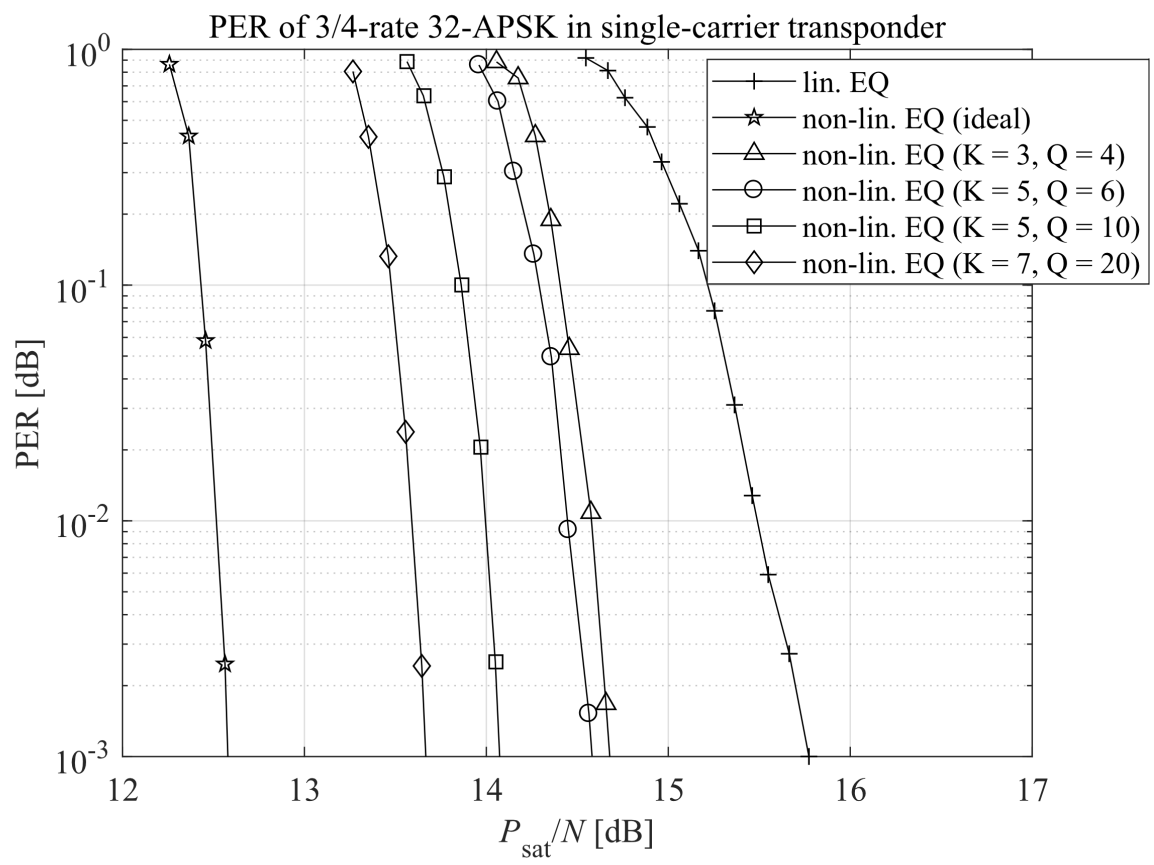

Figure 6. PER of 32-APSK with 3/4-rate LDPC code in a single-carrier 34-Mbaud 5\%-roll-off signal in $36-\mathrm{MHz}$ forward link transponder. Standard linear equalization is compared with 2 iterations of non-linear equalization.

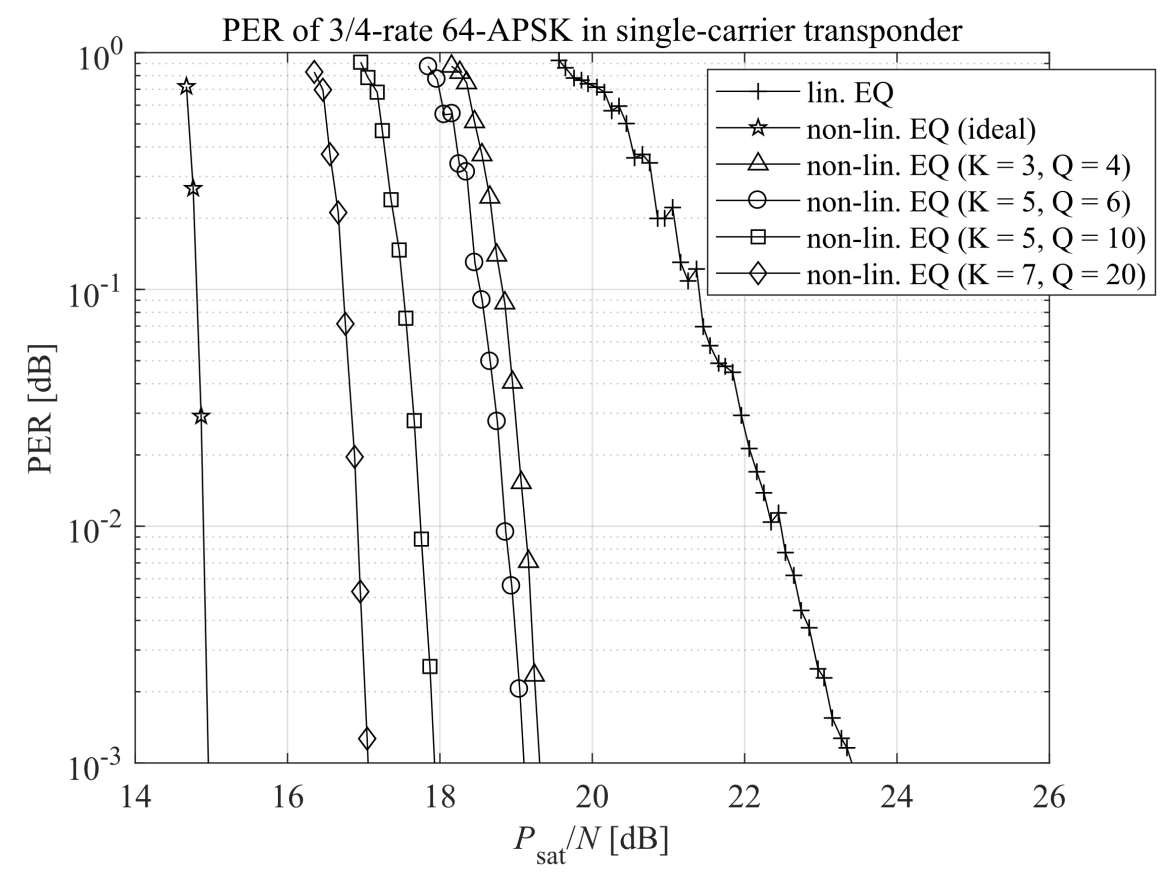

Figure 7. PER of 64-APSK with 3/4-rate LDPC code in a single-carrier 34-Mbaud 5\%-roll-off signal in $36-\mathrm{MHz}$ forward link transponder. Standard linear equalization is compared with 2 iterations of non-linear equalization. 


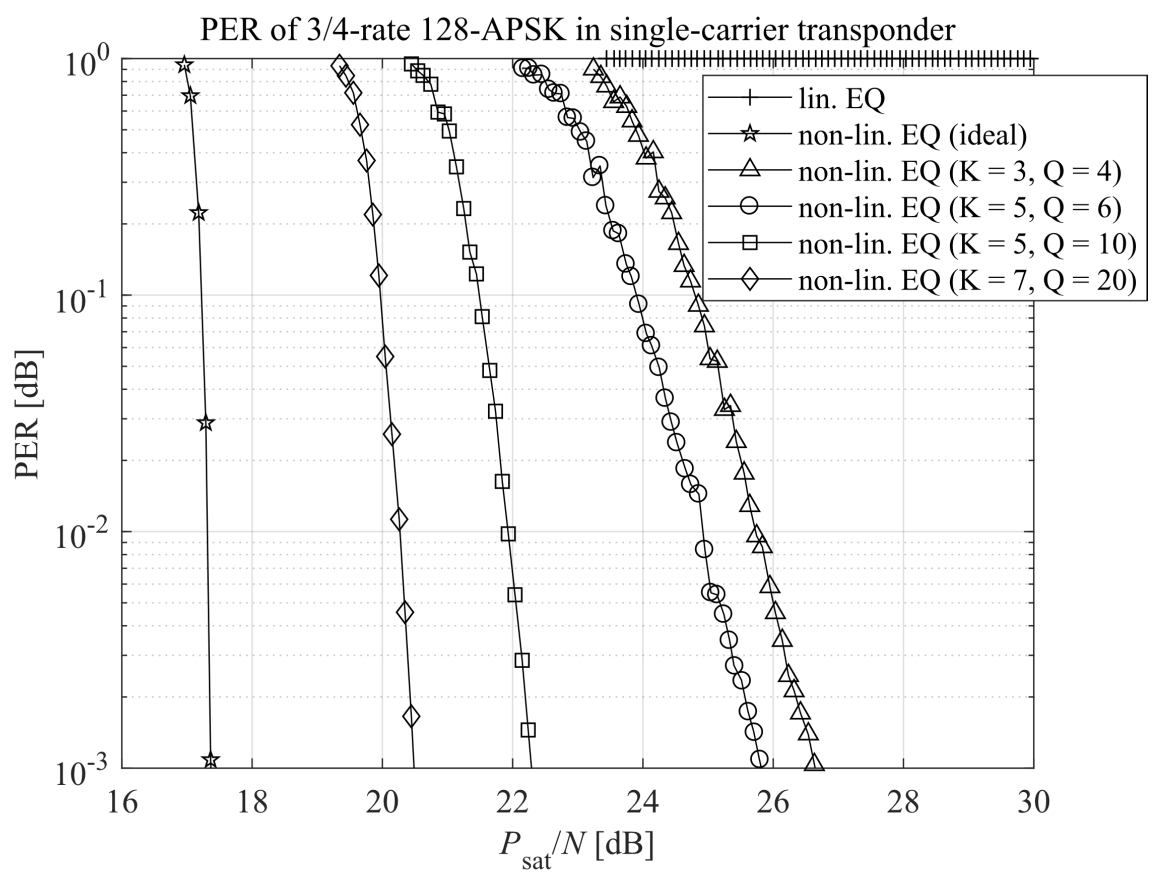

Figure 8. PER of 128-APSK with 3/4-rate LDPC code in a single-carrier 34-Mbaud 5\%-roll-off signal in $36-\mathrm{MHz}$ forward link transponder. Standard linear equalization is compared with 2 iterations of non-linear equalization.

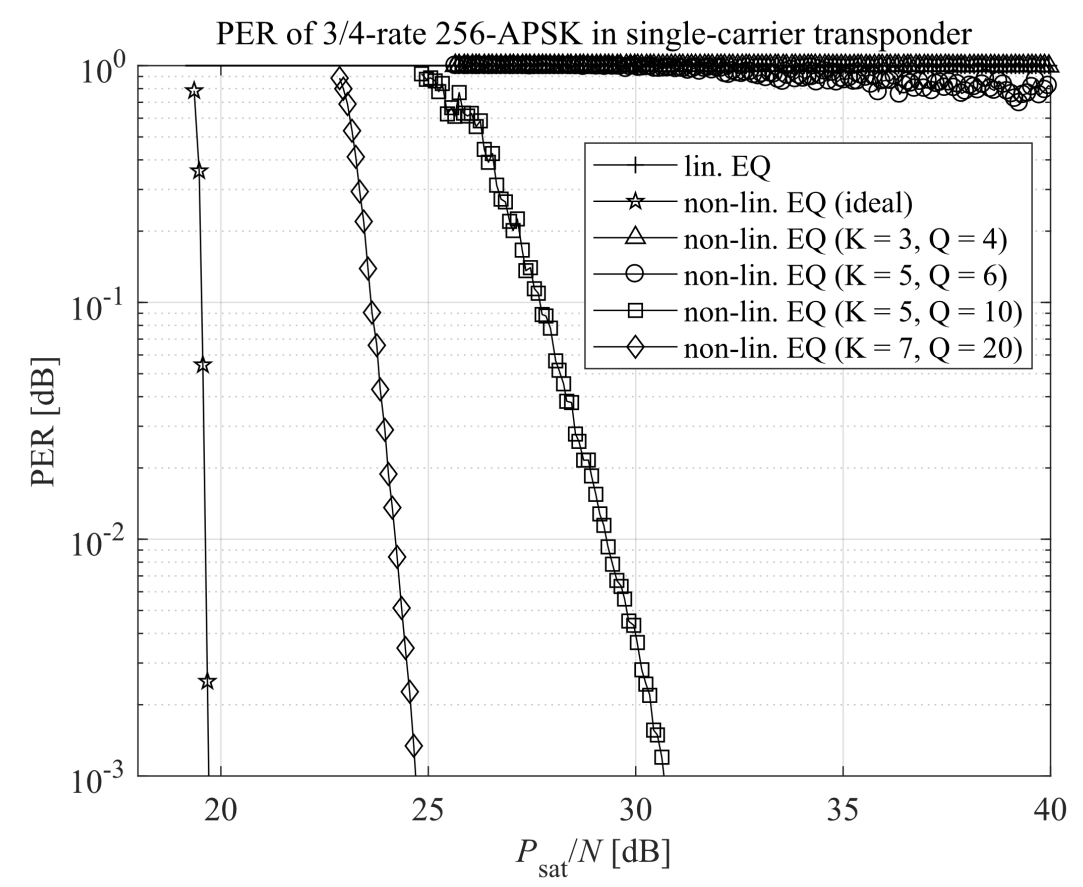

Figure 9. PER of 256-APSK with 3/4-rate LDPC code in a single-carrier 34-Mbaud 5\%-roll-off signal in $36-\mathrm{MHz}$ forward link transponder. Standard linear equalization is compared with 2 iterations of non-linear equalization. 
Therefore, the computational complexity of the non-linear equalizer using the memory polynomial model for channel estimation and distortion cancellation is dominated by (8). Therefore, the receiver complexity remains linear with the size of the model, $(Q+1)(K+1) / 2$, for a given number of iterations [11]. Given the fact that a small-size memory polynomial model with $Q=10$ and $K=5$ already provides sufficient performance in the non-linear channel with memory, this implementation of the non-linear equalizer further reduces the complexity in comparison to the implementation with perfect channel knowledge at the receiver. As a result, low-cost receiver devices.

\section{CONCLUSION}

In this paper, the symbol-based equalizer with non-linear distortion cancellation has been analyzed in a practical setup, including channel estimation at the receiver by means of a memory polynomial model. The improved receiver is particularly suitable for application with wide-band carriers, low roll-off factors, high symbol baud rates, and high-order modulations. The PER performance of the receiver has been assessed for a single-carrier 36-MHz transponder with 34-MBaud symbol rate and $5 \%$ roll-off, similar to DTH broadcasting, using a 3/4-rate LDPC code and modulation orders of 16-APSK, 32-APSK, 64-APSK, 128-APSK, and 256-APSK. Varying the memory depth and the non-linear order of the memory polynomial model, the PER performance of the non-linear equalizer is studied against the standard fractionally-space adaptive linear equalizer, as well as to a setup of the non-linear equalizer with knowledge of the characteristics of the IMUX/OMUX filters and the TWTA at the receiver, in order to evaluate the trade-off between PER performance and complexity. The practical receiver is shown to consistently approach the PER performance of the setup with ideal channel knowledge when increasing the memory depth and the non-linear order. While the standard fractionally-spaced adaptive linear equalizer saturates the PER performance beyond 64-APSK, the non-linear equalizer not only achieves significant energy efficiency gains up to $5.48 \mathrm{~dB}$ for 64 APSK, but also enables the use of higher-order modulation like 128-APSK and 256-APSK, using a memory polynomial model with memory depth of 10 and non-linear order of 5 . The significant energy and spectral efficiency gains promote the proposed equalizer as an effective solution at the receiver in the non-linear satellite channel with memory, enabling the increase of both user rates and system capacity.

\section{ACKNOWLEDGEMENT}

This work has been supported by the German Federal Ministry for Education and Research under contract 16KIS0740. Responsibility for the contents of this publication lies with the author. (Das diesem Bericht zugrundeliegende Vorhaben wurde mit Mitteln des Bundesministeriums für Bildung und Forschung unter dem Förderkennzeichen 16KIS0740 gefördert. Die Verantwortung für den Inhalt dieser Veröffentlichung liegt beim Autor.)

\section{REFERENCES}

1. Second generation framing structure, channel coding and modulation systems for broadcasting, interactive services, news gathering and other broadband satellite applications; part ii: S2-extensions (dvb-s2x) Oct 2014. 
2. Implementation guidelines for the second generation system for broadcasting, interactive services, news gathering and other broadband satellite applications; part ii: S2-extensions (dvb-s2x) Mar 2015.

3. Karam G, Sari H. Analysis of predistortion, equalization, and ISI cancellation techniques in digital radio systems with nonlinear transmit amplifiers. IEEE Trans. Commun. Dec 1989; 37(12):1245-1253.

4. Yu C, Guan L, Zhu E, Zhu A. Band-limited Volterra series-based digital predistortion for wideband RF power amplifiers. IEEE Trans. Microw. Theory Techn. Dec 2012; 60(12):4198-4208.

5. Kelly N, Allegue-Martinez M, Arapoglou PD, Zhu A. Bandwidth-constrained digital pre-distortion technique for multi-carrier satellite communications. Int. J. Satellite Commun. Netw. Apr 2015; .

6. Casini E, De Gaudenzi R, Ginesi A. DVB-S2 modem algorithms design and performance over typical satellite channels. Int. J. Satellite Commun. Netw. Jun 2004; 22:281-318.

7. Ding L, Zhou GT, Morgan DR, Ma Z, Kenney JS, Kim J, Giardina CR. A robust digital baseband predistorter constructed using memory polynomials. IEEE Trans. Commun. Jan 2004; 52(1):159-165.

8. Zhou D, DeBrunner VE. Novel adaptive nonlinear predistorters based on the direct learning algorithm. IEEE Trans. on Signal Proces. Jan 2007; 55(1):120-133.

9. Karam G, Sari H. A data pre-distortion technique with memory for QAM radio systems. IEEE Trans. Commun. Aug 1991; 39(2):336-344.

10. Piazza R, Bhavani Shankar MR, Ottersten B. Data Predistortion for Multicarrier Satellite Channels Based on Direct Learning. IEEE Trans. Signal Proces. Nov 2014; 62(22):5868-5880.

11. Dimitrov S. Non-Linear Distortion Cancellation and Symbol-Based Equalization in Satellite Forward Links. IEEE Trans. Wireless Commun. 2017; 16(7):4489-4502.

12. Proakis J. Digital Communications. 4th edn., McGraw Hill, 2000.

13. Ampeliotis D, Rontogiannis AA, Berberidis K, Papaleo M, Corazza GE. Turbo equalization of non-linear satellite channels using soft interference cancellation. Proc. 4th Advanced Satellite Mobile Systems Conference (ASMS2008), Bologna, Italy, 2008; 289-292.

14. Dimitrov S. Iterative cancellation of non-linear distortion noise in digital communication systems. IEEE Trans. Commun. Jun 2015; 63(6):2325-2336.

15. Biglieri E, Barberis S, Catena M. Analysis and compensation of nonlinearities in digital transmission systems. IEEE J. Sel. Areas Commun. Jan 1988; 6(1):42-51.

16. Kim J, Konstantinou K. Digital predistortion of wideband signals based on power amplifier model with memory. Electron. Lett. Nov 2001; 37:1417-1418.

17. Dimitrov S. Non-linear distortion noise cancellation for satellite return links. Proc. IEEE International Conference on Communications (IEEE ICC 2016), Kuala Lumpur, Malaysia, 2016.

18. Dimitrov S. Non-linear distortion noise cancellation for satellite forward links. Proc. 8th Advanced Satellite Multimedia Systems Conference (ASMS2016), Palma de Mallorca, Spain, 2016. 